\title{
GENERALISATION AND EVALUATION OF MACROSCOPIC MODELS FOR MICROWAVE SUSCEPTORS IN CONTACT WITH HEATED FOODS
}

\author{
M. Celuch ${ }^{1}$, K. Wilczynski², M. Olszewska-Placha ${ }^{1}$, \\ ${ }^{1}$ QWED Sp. z o.o., Krzywickiego 12/1, 02-078 Warsaw, Poland \\ ${ }^{2}$ Faculty of Physics, Warsaw University of Technology, Koszykowa 75, 00-662 Warsaw, \\ Poland \\ mceluch@qwed.eu
}

Keywords: microwave susceptors, microwave heating, electromagnetic modelling, conformal FDTD method, active packaging

\begin{abstract}
The aim of this paper is to evaluate the accuracy of macroscopic models of thin conductive layers used for electromagnetic modelling of microwave susceptors. The equivalent model assumes using a surrogate layer made of dielectric of higher thickness and maintained value of surface resistance. Such an approach allows decreasing computer effort required for electromagnetic analysis with $3 \mathrm{D}$ discrete methods. The present work extends to real-life scenarios, wherein a thin microwave susceptor is placed below food. Evaluation of the macroscopic model accuracy and limits of application is conducted with the aid of analytical calculations and conformal finite difference time domain (FDTD) simulations. The results of the analysis are generalised to give practical guidelines for microwave oven and food packaging designers and manufacturers.
\end{abstract}

\section{Motivation and background}

Thin conductive layers are widely encountered in microwave technology. An example of a daily life application are susceptors in microwaveable food packages [1], [2], [3], which are used in order to increase power dissipation and heating efficiency in food. Moreover, conductive layers (e.g. based on silver nano-particles) are present as the coating of Carbon Fiber Reinforced Polymers (CFRPs) [4]. Those kinds of coatings do not increase the weight of vehicles, therefore they are very useful for aircraft industry and enable to reduce fuel consumption, as well as increase lightning damage protection.

Electromagnetic modelling has been recently recognised by microwave oven and food packaging designers and manufacturers as an important stage of the design process. The most convenient and popular methods for virtual design are finite difference time domain (FDTD) [5] and finite element (FEM) [6] methods. However, introducing a thin conductive layer such as a microwave susceptor, with thickness (below 1 um [7], [8]) by orders of magnitude less than the operating frequency of a microwave oven, into a finite-mesh (as inherent in e.g. FDTD and FEM) typically requires a dedicated equivalent macroscopic model allowing for computationally effective and accurate electromagnetic (EM) and thermal simulations.

Thin conductive layers, characterised by their surface resistance $\left(R_{s}\right)$, are adequately represented with a dielectric surrogate layer of higher thickness and proportionally scaled conductivity, maintaining the value of $R_{s}$ :

$$
R_{s}=\left(d_{0} \sigma_{0}\right)^{-1}=(d \sigma)^{-1},
$$


where $\sigma 0, d_{0}$ stand for values of the original metal and $\sigma, d$ are the scaled parameters of the dielectric surrogate. Systematic evaluation of macroscopic models of microwave susceptors used for enhancing the heating efficiency of microwavable food packages has been reported in [9]. Our studies therein have focused on validity, accuracy, and practical application limits of the proposed macroscopic models of thin metallic layers, in terms of power dissipated in susceptor placed in free space and irradiated by EM wave, at all angles of incidence. In this work we extend our studies to real-life simulation scenarios, in which a microwave susceptor is in contact with food. The discussion is supported with both analytical and numerical conformal FDTD calculations. The accuracy and application limit of the macroscopic model are investigated for all incidence angles and both, TE and TM polarisations of the impinging EM wave, for real foods.

\section{Power dissipation in a surrogate without food}

In Fig. 1 we provide 2D maps of relative power dissipated in a surrogate layer for exemplary values of sheet resistance. The values have been chosen to cover a wide spectrum of applications of thin conductive layers: $1 \Omega$ - absorbing panels, $35 \& 105 \Omega$ - popcorn and pizza susceptors at room temperature, $400 \Omega$ - food susceptors breaking at above $240 \mathrm{deg}$, $20000 \Omega$ - susceptors after use. The results of this study are obtained analytically with the use of equations, which are discussed in detail in Section III.

Fig. 2 shows vertical cuts through the maps of Fig. 1, i.e., the dependence of relative power dissipated in a surrogate on the surrogate's thickness. Figs. 1 and 2 indicate that the values of $\mathrm{Rs}=30 \div 400 \Omega$ enable the best absorption for most field patterns (provided that the susceptor is in free space). This is further confirmed by the continuous plots of relative power dissipated in an ultra-thin susceptor, as a function of the susceptor's sheet resistance, presented in Fig. 3.

Note that analytical calculations indicating $\mathrm{Rs}=30 \div 400 \Omega$ as the optimum range for microwave heating have been reported earlier [1], however, those calculations were limited to normal incidence (TEM waves). Our study extends the proof to oblique incidence. It also shows that for higher incidence angles, TM waves heat more efficiently lower-resistance susceptors, while TE waves heat more efficiently higher-resistance susceptors. These observations are complementary to the investigation of equivalent angles of incidence in multi-modal domestic ovens, and thereby can help design of microwaveable food packages.

From the viewpoint of macroscopic surrogates, the results provided in Figs. 1 and 2 clearly show the range of admissible scaling of the surrogate thickness. The scaling remains neutral to power dissipation, provided that the scaled surrogate thickness does not exceed $1 \mathrm{~mm}$, which at $2.45 \mathrm{GHz}$ corresponds to ca. 0.05 wavelength. In [9], the analytical results were further validated with conformal FDTD simulations using QuickWave 3D software [11]. 


$$
1-\left|\mathrm{S}_{11}\right|^{2}-\left|\mathrm{S}_{21}\right|^{2} @ \mathrm{f}=2.45 \mathrm{GHz} \quad \epsilon_{\text {food }}=1, \sigma_{\text {food }}=0
$$
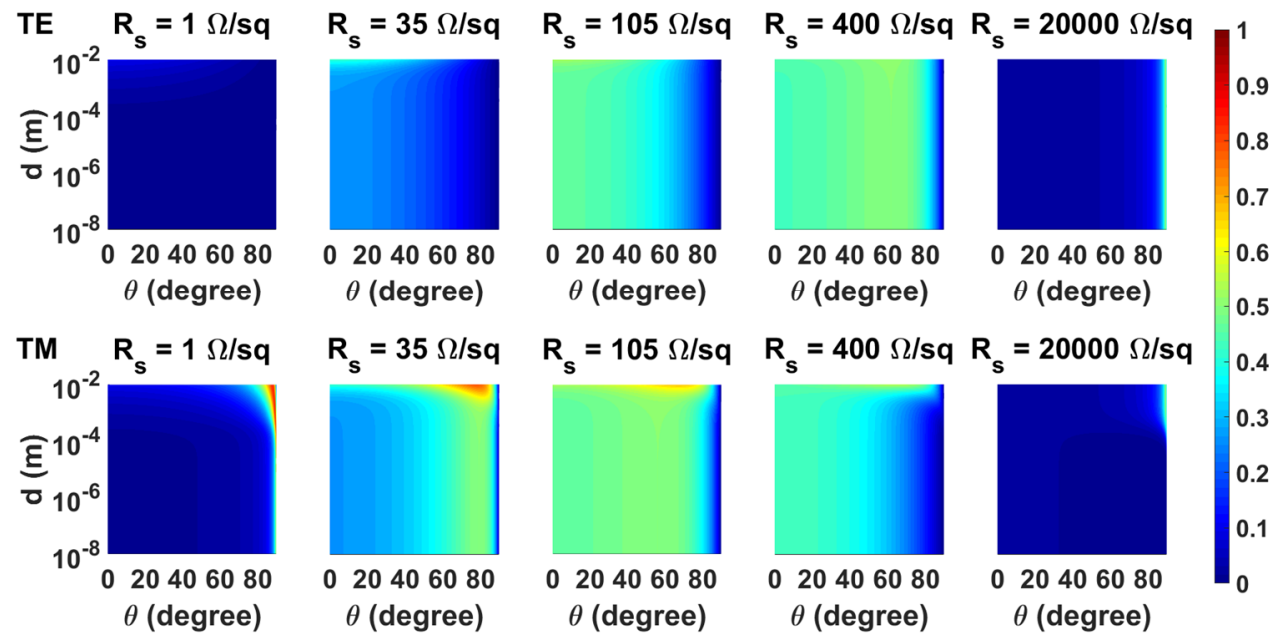

Fig. 1. Maps of relative dissipated power $\left(1-\left|S_{11}\right|^{2}-\left|S_{21}\right|^{2}\right)$ in a susceptor in free space (without food) for varying angles of incidence $\theta$ and thickness $d$ of the surrogate.
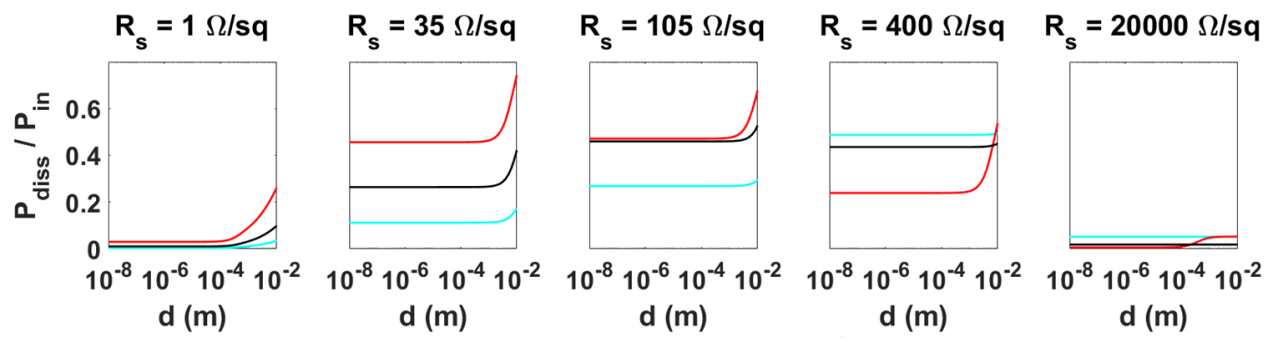

$$
\text { - TE }\left(70^{\circ}\right) \text { - TM }\left(70^{\circ}\right) \text { - TEM }
$$

Fig. 2. Vertical cuts through the maps of Fig. 1, for normal incidence (TEM), transverse electric (TE) and transverse magnetic (TM) polarisation at $70 \mathrm{deg}$.
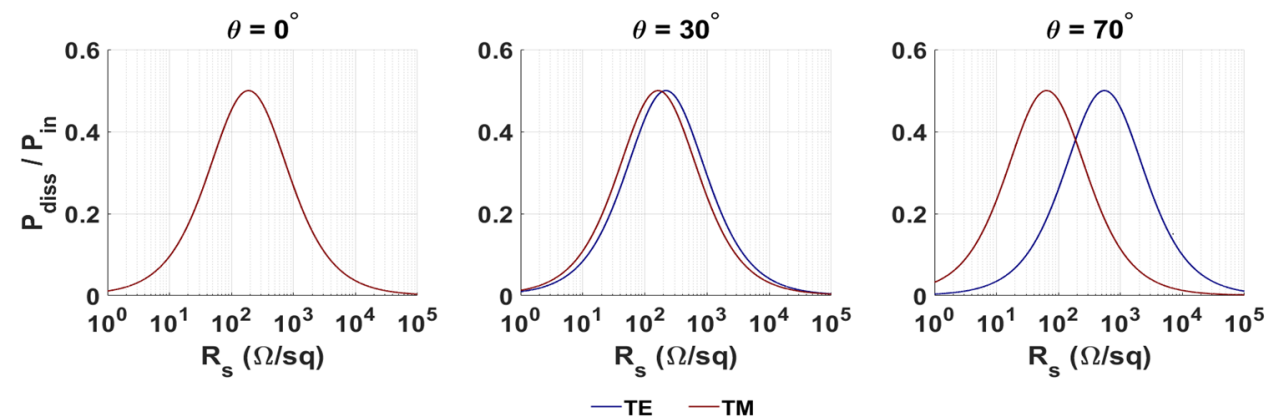

Fig. 3. Relative dissipated power in a susceptor placed in free space and irradiated at different angles, by waves of different polarisations. 


\section{Power dissipation jointly in the surrogate and food}

We now extend our study to the case of a susceptor being in contact with a slice of food, which is a typical situation in microwaveable food packages. The stacked structure as in Fig. 4, illuminated with EM wave at varying angle of incidence $\theta$ is considered. In the model, susceptor and food are assumed to have constant and isotropic values of permittivity and conductivity and the food is made of a non-magnetic material.

The electromagnetic multi-layered scenario shown on the left of Fig. 4 can be conveniently analysed with the transmission line theory, where it reduces to the flow graph shown on the right of Fig. 4. The graph accounts for three impedance steps at media boundaries, which the EM wave faces when propagating through the structure.

The system of Fig. 5 can be reduced to a two-port network, described with the S-matrix:

$$
\begin{aligned}
S_{11}= & \Gamma_{A B}+T_{A B} T_{B A} \cdot \tau_{B}^{2} \cdot\left(\Gamma_{B C}+\Gamma_{C D} \cdot \tau_{C}^{2}\right) \cdot \Delta, \\
S_{22}= & \Gamma_{D C}+T_{C D} T_{D C} \cdot \tau_{C}^{2} \cdot\left(\Gamma_{C B}+\Gamma_{B A} \cdot \tau_{B}^{2}\right) \cdot \Delta, \\
& S_{21}=T_{A B} T_{B C} T_{C D} \cdot \tau_{B} \tau_{C} \cdot \Delta, \\
& S_{12}=T_{D C} T_{C B} T_{B A} \cdot \tau_{B} \tau_{C} \cdot \Delta,
\end{aligned}
$$

where:

$$
\Delta=\left(1-\Gamma_{B A} \Gamma_{B C} \cdot \tau_{B}^{2}-\Gamma_{C B} \Gamma_{C D} \cdot \tau_{C}^{2}-\Gamma_{B A} \Gamma_{C D} \cdot \tau_{B}^{2} \tau_{C}^{2}\right)^{-1},
$$

$\Gamma_{i j}$ and $T_{i j}$ parameters stand for reflection and transmission coefficients (for wave amplitudes) respectively from $i$-th to $j$-th layer (where $i, j$ stand for $A-D$ ):

$$
\Gamma_{i j}=\left\{\begin{array}{l}
\frac{z_{j}-Z_{i}}{Z_{i}+Z_{j}}, \text { for TEM } \\
\frac{z_{j} \cos \theta_{i}-Z_{i} \cos \theta_{j}}{Z_{j} \cos \theta_{i}+Z_{i} \cos \theta_{j}}, \text { for TE } \\
\frac{Z_{j} \cos \theta_{j}-Z_{i} \cos \theta_{i}}{Z_{i} \cos \theta_{i}+Z_{j} \cos \theta_{j}}, \text { for TM }
\end{array} \quad T_{i j}=\left\{\begin{array}{l}
\frac{2 Z_{j}}{z_{i}+Z_{j}}, \text { for TEM } \\
\frac{2 Z_{j} \cos \theta_{i}}{Z_{j} \cos \theta_{i}+Z_{i} \cos \theta_{j}}, \text { for TE } \\
\frac{2 z_{j} \cos \theta_{i}}{z_{i} \cos \theta_{i}+Z_{j} \cos \theta_{j}}, \text { for TM }
\end{array}\right.\right.
$$

and $\tau_{i}$ stands for a wave transmission coefficient through $i$-th layer:

$$
\tau_{i}=e^{-\gamma_{i} \cdot d_{i} \cdot \cos \theta_{i}}
$$

In above equations $Z_{i}=\sqrt{\frac{\mu_{i}}{\varepsilon_{i}}}$ is a wave impedance of the material in $i$-th layer, $\gamma_{i}=$ $2 \pi j f \sqrt{\mu_{i} \varepsilon_{i}}$ is a propagation constant at the frequency of $f, d_{i}$ stands for the thickness of the layer, and $\cos \theta_{i}$ is defined as follows:

$$
\cos \theta_{i}=\sqrt{1-\left(\mu_{i} \varepsilon_{i} c^{2}\right)^{-1} \sin ^{2} \theta_{0}},
$$

where $\theta_{0}$ stands for an incidence angle in air and $c$ for speed of light. In the above equations $\mu_{i}$ and $\varepsilon_{i}$ stand for complex permeability and permittivity of material in $i$-th layer, which for the purpose of this paper are defined as $\mu_{i}=\mu_{0}$ and $\varepsilon_{i}=\varepsilon_{r i} \varepsilon_{0}-\frac{j \sigma_{i}}{2 \pi f}$. Index $i \equiv 0$ denotes vacuum parameters. 


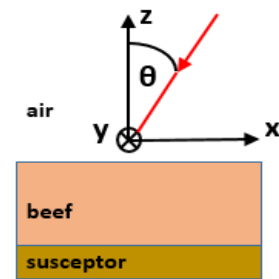

air

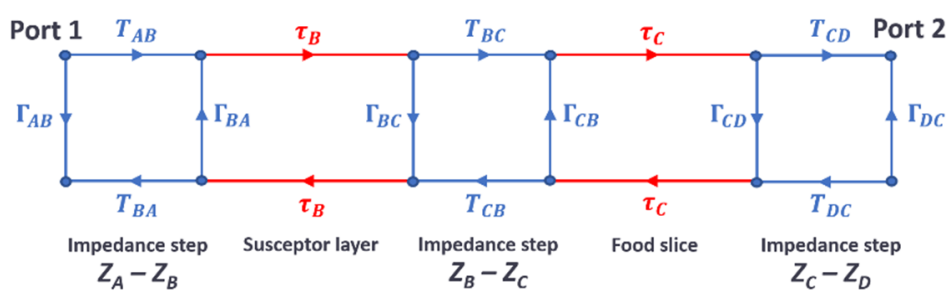

Fig. 4. Four-layer EM model (left) and (right) its microwave flow graph: A and D layers stand for air, B for a susceptor, and C for a food slice.

Based on the above analysis and equations, total relative power dissipated, jointly in a susceptor and $1 \mathrm{~cm}$-thick slice of beef $\left(\varepsilon_{r}=48.2, \sigma=2.194 \mathrm{~S} / \mathrm{m}\right.$ at room temperature), defined as $\left(1-\left|S_{12}\right|^{2}-\left|S_{22}\right|^{2}\right)$, is calculated for varying thicknesses of the dielectric surrogate and angles of incidence. The results are presented in Fig. 5 in a form of 2D maps. It is clearly seen that using a surrogate layer of thickness up to $1 \mathrm{~mm}$ does not affect the dissipated power, compared to the original ultra-thin susceptor. Thereby, the practical limit of application of the macroscopic model of thin conductive sheets derived in [9] for susceptors in free space remains valid for real-life scenarios, in which susceptor is in contact with food. The results further prove that the heating will be more efficient for TM than for TE-waves, and the highest absorption is obtained for high angles of TM wave incidence.

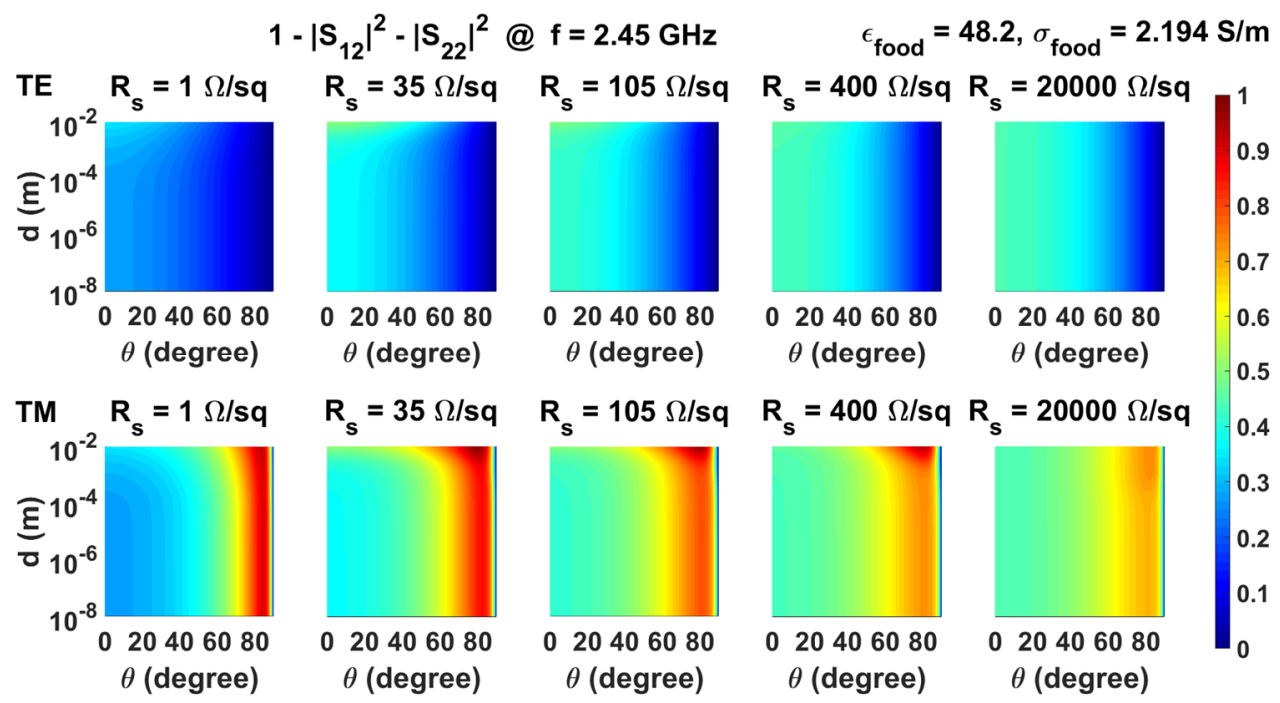

Fig. 5. Maps of total relative power dissipated jointly in a susceptor and $1 \mathrm{~cm}$-thick slice of beef $(\varepsilon=48.2 \sigma=2.194 \mathrm{~S} / \mathrm{m})$, for EM waves incident at varying angle $\theta$ from air onto beef, and varying thickness $d$ of the surrogate. 


\section{Power dissipated separately in the surrogate and the food}

In this section we extend the analysis of the scenario of Fig. 4, by considering power dissipated in each layer of the model. We consider power transmitted through an arbitrary plane parallel to the layers:

$$
P=\operatorname{Re}\left(\boldsymbol{E} \times \boldsymbol{H}^{*}\right) \cdot \hat{\mathbf{z}}=\left(\frac{\cos \theta}{Z}\right)^{*} \cdot\left(E^{+}+E^{-}\right) \cdot\left(E^{+}-E^{-}\right)^{*},
$$

where $\boldsymbol{E}, \boldsymbol{H}$ stand for electric and magnetic fields in the plane, $\hat{\boldsymbol{z}}$ is a unit vector normal to the layers, $\cos \theta$ was defined with equation (8), $Z$ is impedance, $E^{+}$and $E^{-}$stand for complex amplitudes of electric field of the incident and reflected waves at the considered plane. Power dissipated in $i$-th layer can be calculated as a difference of power transmitted through the opposite planes of the layer:

$$
P_{i}=P_{i}^{\text {in }}-P_{i}^{\text {out }}
$$

For EM wave incident at port 1, electric field amplitudes in layer B (see Fig. 5) can be calculated as follows:

$$
\begin{array}{ll}
E_{\text {in }}^{+}=\frac{1}{T_{B A}}\left(1+\Gamma_{B A} S_{11}\right) \cdot E_{0}^{+}, & E_{\text {out }}^{+}=E_{\text {in }}^{+} \cdot \tau_{B}, \\
E_{\text {in }}^{-}=\frac{1}{T_{B A}}\left(-\Gamma_{A B}+S_{11}\right) \cdot E_{0}^{+}, & E_{\text {out }}^{-}=E_{\text {in }}^{-} \cdot \tau_{B}^{-1},
\end{array}
$$

and in layer $\mathrm{C}$ (further from the incident port):

$$
\begin{gathered}
E_{\text {out }}^{+}=\frac{1}{T_{C D}} S_{21} \cdot E_{0}^{+}, \quad E_{\text {in }}^{+}=E_{\text {out }}^{+} \cdot \tau_{C}^{-1}, \\
E_{\text {out }}^{-}=\Gamma_{C D} \cdot E_{\text {out }}^{+}, \quad E_{\text {in }}^{-}=E_{\text {out }}^{-} \cdot \tau_{C},
\end{gathered}
$$

where $E_{0}^{+}$stands for an amplitude of an incident wave at port 1. Analogical equations can be derived in the case of a wave incident at port 2 , if one reverts indices of layers and ports.

The above conducted analysis and formulated equations are confirmed with the use of conformal FDTD modelling performed in QuickWave 3D software [11]. In order to simulate normal incidence (TEM) of EM wave on the considered multi-layer stacked structure (Fig. 4), a parallel-plate line model is used. For the purpose of modelling plane wave with an arbitrary incidence angle, we use a rectangular waveguide with a square cross section $a \times a$ supporting TE11 and TM11 modes. In order to obtain an arbitrary angle $\theta$ of EM wave incidence in air, the waveguide dimension $a$ needs to be adjusted following the formula:

$$
a=\frac{c}{f \cdot \sin \theta \cdot \sqrt{2}},
$$

where $c$ stands for the speed of light in vacuum.

Standard stair-case formulation of the FDTD method requires that the surrogate thickness occupies at least one full FDTD cell, which for thin layers significantly increases simulation time as the FDTD time step decreases. QuickWave 3D software implements conformal FDTD, which allows for adopting thin layers filling only a part of the FDTD cell, thereby alleviating the stair-case limitation and achieving the geometric flexibility classically attributed to FEM formulations. In the conducted EM analysis, FDTD cell size in the direction of wave propagation is set to $1 \mathrm{~mm}$ and $10 \mu \mathrm{m}$-thick surrogate is considered. 
Fig. 6 presents comparison of analytical and conformal FDTD results for dissipated power, calculated separately for the susceptor and raw beef, as a function of surface resistance of the susceptor. Analytical data are fully consistent with the electromagnetic simulation results. It is seen that the absorption in susceptor remains maximal for its sheet resistance in the range of $\mathrm{Rs}=30 \div 400 \Omega$, similarly as for the susceptor in free space. However, total value of dissipated power tends to increase monotonously with the increasing sheet resistance of the susceptor (except for very high incidence angle and TM polarisation, where a subtle optimum occurs around the optimum of the susceptor heating). Two physical effects are therefore observed, when the surface resistance of the susceptor increases: first, the susceptor heats more and then, the susceptor stops heating but its backing of the food causes more efficient volumetric heating of the food itself.

Note that in real-life microwaveable food packages, the susceptors are manufactured so that they break or otherwise increase their surface resistance during the heating [1], [10]. Our graphs in Fig. 6 help therefore explain how the initial surface heating by the susceptor is followed by the volumetric heating of the actual load; in typical microwaveable foods the volumetric heating phase is further enforced by the increase of food losses with temperature, in typical foods [10].

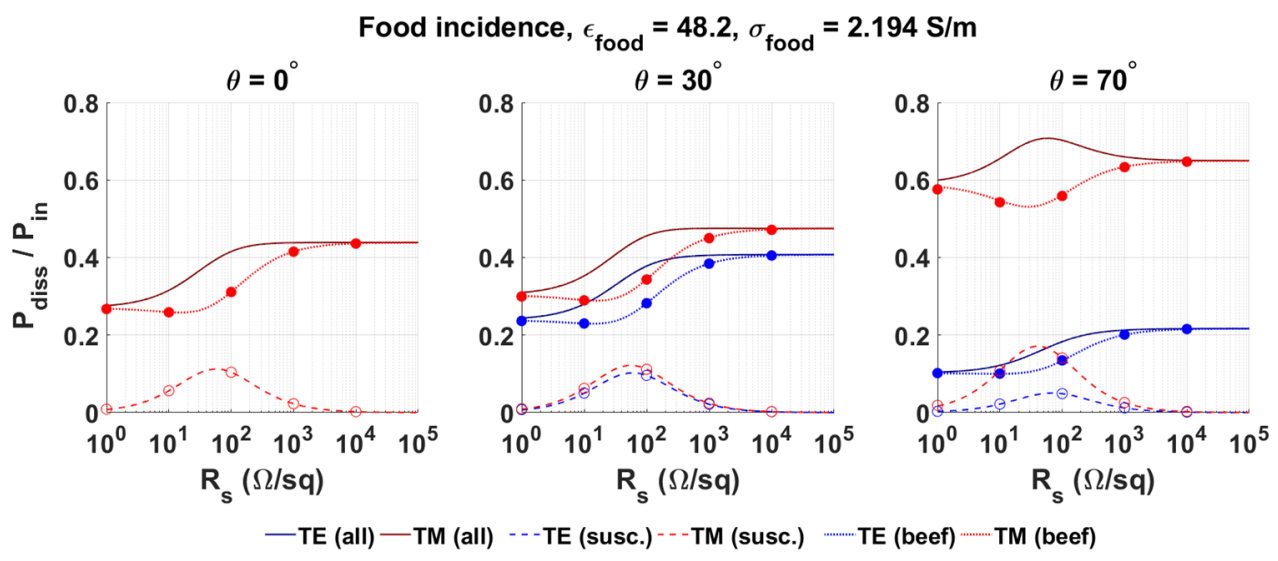

Fig. 6. Relative power dissipated separately in $10 \mu \mathrm{m}$ susceptor (susc) and $1 \mathrm{~cm}$ raw beef (beef) layers; and jointly (all) in both layers, for EM waves incident from air onto food backed by the susceptor. Analytical results are plotted by lines; dots and circles indicate conformal FDTD results for food and susceptor, respectively.

\section{Conclusions}

In the present work an application of macroscopic models in a form of dielectric surrogate layers of significantly higher thickness for microwave susceptors analysis is considered. In extension to earlier studies, the discussion addresses real-life scenarios, where a microwave susceptor is in contact with food, as it typical microwaveable food packages. The evaluation of the macroscopic model is conducted with the aid of analytical calculations and conformal FDTD simulations, accounting for various angles of EM wave incidence and wave polarisation, surface resistance of microwave susceptor, and surrogate thickness. The systematic evaluation of the equivalent macroscopic model in terms of power dissipated in the structure allows to derive a practical limit of $1 \mathrm{~mm}$ surrogate thickness (at $2.45 \mathrm{GHz}$ ), for application of the surrogate model in the modelling scenarios where the susceptor 
remains in contact with food. Moreover, the studies aiming at extracting power dissipated separately in food and susceptor resulted in formulating a range of susceptors' surface resistance assuring the highest power absorption in susceptor, and the highest absorption in the overall package, which constitutes practical guidelines for food packaging designers and manufacturers.

\section{References}

1. M. R. Perry and R. R. Lentz, "Susceptors in microwave packaging," in Development of packaging and products for use in microwave ovens, $1^{\text {st }}$ ed., W. Lorence and P. S. Pesheck, London, United Kingdom: Woodhead Publishing Limited and CRC Press, 2009, 207-236.

2. T. H. Bohrer, "Shielding and field modification - thick metal films," in Development of packaging and products for use in microwave ovens, $1^{\text {st }}$ ed., W. Lorence and P. S. Pesheck, London, United Kingdom: Woodhead Publishing Limited and CRC Press, 2009, 237-266.

3. P. Risman, "Modelling the effects of active packaging of microwave foods," in Development of packaging and products for use in microwave ovens, $1^{\text {st }}$ ed., W. Lorence and P. S. Pesheck, London, United Kingdom: Woodhead Publishing Limited and CRC Press, 2009, 349-371.

4. Ha, M. S., Kwon, O. Y., Choi, H. S., Composites Research, 2010, 23, 31-36.

5. A. Taflove, M. Celuch-Marcysiak, and S. Hagness, "Local Subcell Models of Fine Geometrical Features," in Computational Electrodynamics - The Finite-Difference Time-Domain Method, $3^{\text {rd }}$ Edition, A. Taflove and S. Hagness, Boston-London: Artech House, 2005, pp. 407-462.

6. P. P. Silvester and R. L. Ferrari, Finite Elements for Electrical Engineers, $3^{\text {rd }}$ Ed., Cambridge University Press, 1996.

7. W. Gwarek, "Modeling and measurements of susceptors for microwave heating applications", at: IEEE IMS Workshop "Recent Advances in Microwave Power Applications and Techniques RAMPAnT”, Boston, June 2009.

8. Krupka, J., Gwarek, W., "Measurements and modeling of planar metal film patterns deposited on dielectric substrates",IEEE Microwave and Wireless Components Letters, 2009, 19, 134-136.

9. M. Celuch, K. Wilczynski, M. Olszewska-Placha, "Macroscopic Models of Thin Conductive Layers: Systematic Evaluation for Microwave Heating and Shielding Applications", IEEE International Microwave Symposium, Boston, May 2019, 4750 .

10. P. O. Risman, "Microwave oven and food compatibility: historical background, the present situation and future possibilities" private communication; also presented at: 42 $2^{\text {nd }}$ Annual Microwave Power Symp. (IMPI), New Orleans, LA, June 2008.

11. The QuickWave EM Software (1997-2019) website. [Online] Available: http://www.qwed.eu 